\title{
Uniportal video-assisted thoracoscopic surgical (VATS) segmentectomy with preoperative dual localization: right upper lobe wedge resection and left upper lobe upper division segmentectomy
}

\author{
Kook Nam Han, Hyun Koo Kim, Young Ho Choi \\ Departments of Thoracic and Cardiovascular Surgery, Korea University Guro Hospital, Korea University College of Medicine, Seoul, Korea \\ Correspondence to: Hyun Koo Kim, MD, PhD. Departments of Thoracic and Cardiovascular Surgery, Korea University Guro Hospital, Korea \\ University College of Medicine, 97 Guro-donggil, Guro-gu, Seoul 152-703, Korea. Email: kimhyunkoo@korea.ac.kr.
}

Submitted Nov 02, 2015. Accepted for publication Mar 02, 2016.

doi: 10.21037/acs.2016.03.06

View this article at: http://dx.doi.org/10.21037/acs.2016.03.06

\section{Clinical vignette}

A 75-year-old male presented with abnormalities upon chest computed tomography (CT) scanning at a routine check. He had a 1.7 -cm sized ground glass opacity (GGO) on the posterior segment of the right upper lung (RUL) and a 1.2-cmsized semisolid lung nodule on the left upper lung (LUL). To differentiate synchronous metastasis, sequential CT-guided core biopsy was performed for the GGO lesion on the posterior segment of the RUL and the posterior segment of the LUL. Both lesions were suspected to be adenomatous hyperplasia or non-small cell lung cancer. Adenocarcinoma was detected in situ upon pathologic examination. A positron emission tomography (PET) scan showed no lymph node metastasis or extrathoracic distant metastasis. The LUL semisolid lesion showed mild hypermetabolism, while the RUL pure GGO lesion showed no definite uptake on PET scan. Pulmonary function was as follows: forced vital capacity (FVC), $3.07 \mathrm{~L}$ (75\%); forced expiratory volume in 1 second (FEV1), $2.34 \mathrm{~L}$ (88\%); carbon monoxide lung diffusion capacity (DLCO), $20.7 \mathrm{~mL} / \mathrm{mmHg} / \mathrm{min}$ (116\%). The patient was referred for surgical resection of bilateral synchronous lung lesions. In this case, by employment of the dual localization technique (hook-wire and lipiodol), we performed bilateral uniportal video-assisted thoracoscopic surgery (VATS) resection. Wedge resection was carried out for the GGO lesion on the posterior segment of the RUL and left upper divisional segmentectomy was carried out for the semisolid lesion on the posterior segment of the LUL (Video 1).

\section{Surgical technique}

Preparation and exposition

Preoperative CT-guided dual localization with a hookwire and lipiodol for bilateral lung lesions was performed two hours before surgery. The operation was performed under general anesthesia with double lumen endotracheal tube intubation. The patient was positioned in both lateral decubitus positions with arm elevation for each surgery. All participants in the operating room wore lead aprons during real-time fluoroscopy for localization of the lung lesions. We used a 5-mm diameter 30-degree thoracoscope (Karl Storz, Tuttlingen, Germany) and articulating and curved endoscopic instruments. A wound protector was inserted through the port to protect the port site from contamination in case of lung malignancy and to achieve better instrumental performance (Figure 1). The surgeon always stood on the right side of the patient, regardless of the operation side. Surgical assistants stood on the left side of the patient.

\section{Operation}

Uniportal VATS wedge resection for GGO lesion with guidance by real-time $\mathrm{C}$-arm fluoroscopy

Uniportal right upper lobe wedge resection was initiated by the creation of a $1.5-\mathrm{cm}$ long incision in the right upper 


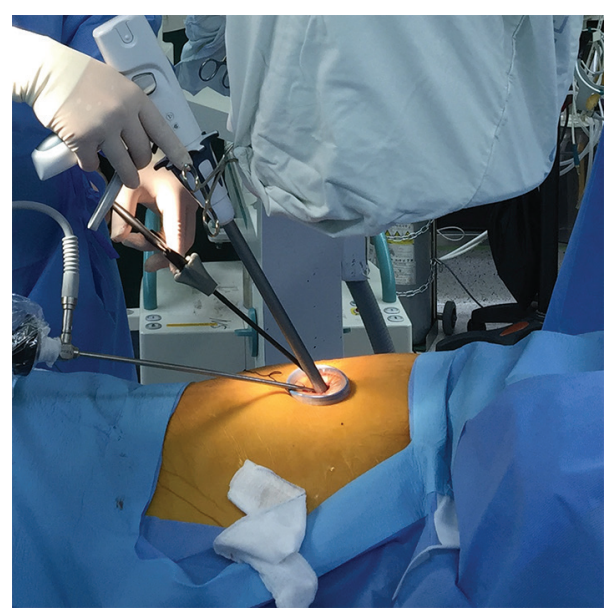

Figure 1 Instrumentation during uniportal video-assisted thoracoscopic surgery (VATS) segmentectomy under real-time $\mathrm{C}$-arm guidance.

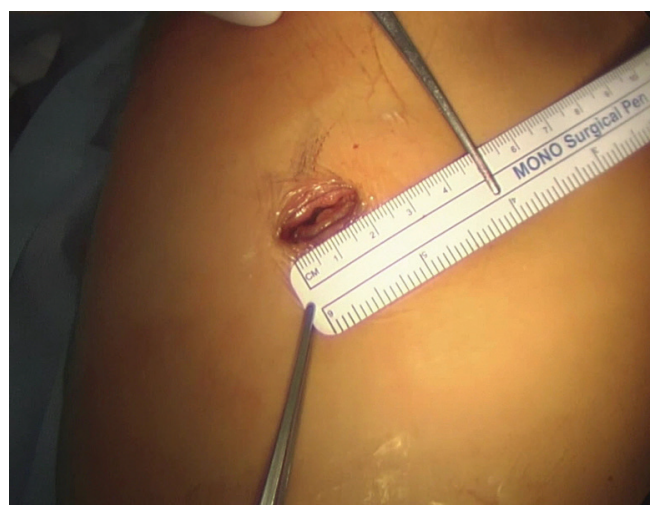

Figure 2 Incision for uniportal video-assisted thoracoscopic surgery (VATS) segmentectomy.

fifth intercostal space, along the anterior axillary line. Thoracoscopic exploration revealed the hook-wire from the pleura localized to the target lung lesion. Dislodgment of the hook-wire is a common event in preoperative localization. Therefore, the advantage of dual localization with a radiopaque contrast media such as barium or lipiodol is a higher detection rate of lung lesions in the case of hookwire dislodgement. The dual localization technique also benefits from correct localization of the target lung lesion with a hook-wire from the pleural surface by thoracoscopy and a sufficient resection margin of more than $2 \mathrm{~cm}$ from the lesion with lipiodol and guidance using real-time C-arm fluoroscopy. After insertion of an intrapleural continuous analgesia infusion system and a 16-French chest drain at the incision site, the wound was closed. The patient was then positioned in the right lateral decubitus position for left upper divisional segmentectomy.

\section{Uniportal VATS LUL divisional segmentectomy}

A 2-cm long incision was created in the fifth intercostal space along the anterior axillary line (Figure 2). The hookwire was easily identified at the LUL lesion. First, the interlobar fissure was dissected to isolate the posterior and lingular segmental branches of the pulmonary artery. Then, the upper divisional trunk of the pulmonary artery could be divided by opening the mediastinal pleura after lung traction posteriorly and further dissection could be performed through the space between the left upper pulmonary artery and vein. Interlobar and segmental lymph nodes could be dissected by careful manipulation. The segmental branches of the upper divisional pulmonary vein were divided more easily after division of the upper divisional branches of the pulmonary artery. To divide the upper divisional segmental plane, intraoperative optic bronchoscopy was used before stapling the segmental bronchus. The intersegmental plane between the lingular and upper divisional segments could be delineated by inflation and deflation using pressure jet ventilation $\left(2 \mathrm{~kg} / \mathrm{cm}^{2}\right)$ of the segmental bronchus. Alternatively, a breath was given to visualize the noninflating segment after occlusion of the target segmental bronchus.

The intersegmental plane was divided along the plane identified by the above procedure and combined use of real-time C-arm fluoroscopy. We performed lobe specific sampling in this case, as there was no lymph node enlargement or uptake upon PET scanning.

\section{Completion}

A chest drain and intrapleural continuous analgesia pump were inserted through the same incision and the wound was closed. The incisional length was $1.5 \mathrm{~cm}$ on the right thorax and $2 \mathrm{~cm}$ on the left thorax (Figure 3). After extubation in the operating room, the patient was transferred to the surgical intensive care unit for postoperative care.

\section{Comments}

\section{Clinical results}

Between March 2012 and June 2015, we performed more 

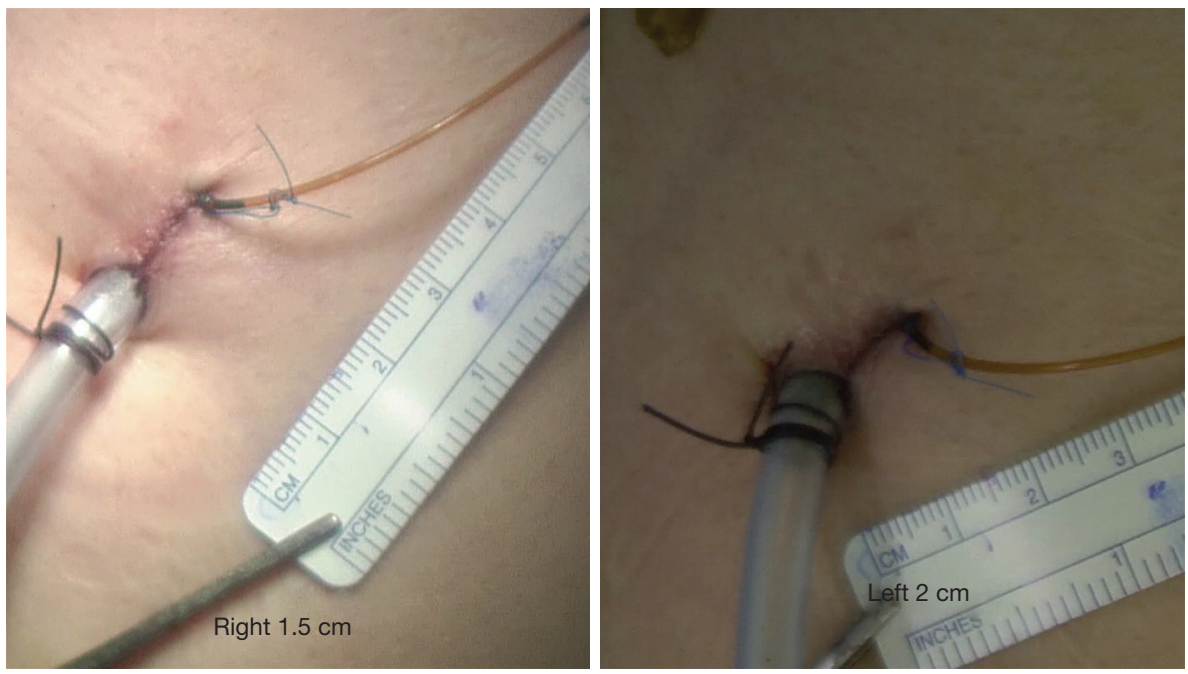

Figure 3 Postoperative wound.

than 300 uniportal VATS major lung resections and 30 of these patients underwent VATS segmentectomy using a uniportal approach. Our indications for uniportal segmentectomy were early lung malignancy (peripheral cT1N0) with a tumor less than $2 \mathrm{~cm}$ in diameter and a GGL that was less than $50 \%$ solid (1). Pulmonary segmentectomy carried out in patients not suitable for wedge resection and those with inflammatory lung disease in order to preserve pulmonary reserve was also included in our series. Of the 30 cases of uniportal segmentectomy, 21 $(70 \%)$ cases had lung malignancy with a mean tumor size of $1.6 \pm 0.5 \mathrm{~cm}$ (range, $0.9-2.7 \mathrm{~cm}$ ). The mean operation time was 147.2 \pm 59.3 minutes (range, 30-236 minutes). Preoperative localization was successful in $14(73.7 \%)$ patients. There was one case of conversion to lobectomy in a patient who had not undergone preoperative localization, because we failed to find the lesion after segmentectomy. There was no lymph node metastasis in any patient upon pathologic examination. There was one case of prolonged air leak ( $>5$ days) and two patients had postoperative pneumonia; they recovered with conservative management. There was one early mortality ( $<30$ days) due to septic shock developing after systemic arterial embolism due to underlying disease. The mean chest tube indwelling time was $4.6 \pm 1.6$ days.

\section{Advantages}

In addition to a smaller incisional scar, as compared to multi-port VATS, the potential benefits of uniportal
VATS segmentectomy are less postoperative intercostal neuralgia via a reduction in the number of ports, a better postoperative course, earlier removal of the chest drain and a shorter hospital stay (2). Chest wall paresthesia can also be reduced by decreasing the number of ports and removing the need for a trocar to reduce intercostal neuralgia. Uniportal VATS segmentectomy may be a better option in elderly patients with poor pulmonary reserve (3). In addition, this approach can be applied in early lung cancer, elderly surgical candidates and patients who require lung preservation such as those undergoing pulmonary metastasectomy for metastases from other malignancies. One-stage bilateral surgery can be performed safely in patients with bilateral lung lesions.

Specifically, in our series, we focused on reducing the incisional length (to $2-3 \mathrm{~cm}$ ) as compared to other case series of uniportal VATS major lung resection (in which incision size is usually $3-5 \mathrm{~cm}$ ). However, based on our experience, a smaller volume of segmentectomy specimen can be removed through $2-3 \mathrm{~cm}$ incisions. This issue should be evaluated by future researchers.

Conversion to lobectomy is prevented with the use of a proper preoperative localization technique such as the use of a hook-wire (4), radiocontrast material or radioisotope or fiducial placement by the recently described electromagnetic navigational bronchoscopy procedure.

\section{Caveats}

Although it has the potential merits mentioned above, 
our surgical approach required a learning curve, even for surgeons experienced in conventional multi-port VATS major lung resection. Reducing the number of ports systematically from three to two to one can help thoracic surgeons to adopt the uniportal VATS procedure (5). With improvement of endoscopic devices, the strategy for the VATS approach might be transitioned to a more minimal incision. Appropriate selection of endoscopic devices and surgical staplers plays an important role when performing uniportal VATS segmentectomy. This can be facilitated by the use of articulating endoscopic instruments, curved devices, vascular clips, energy devices and a high definition camera system with a $3.3-\mathrm{mm}$ diameter endoscope.

More recently, respectable long-term outcomes can be achieved by uniportal VATS segmentectomy of early lung cancer in selected population, which is comparable with conventional multi-port VATS (Diego Gonzalez-Rivas, presented at the European Lung Cancer Conference 2015, unpublished data). Further studies in larger populations and randomized trials are required to adopt our strategy as a first choice of thoracic procedure.

\section{Conclusions}

Our results suggest the safety of uniportal VATS segmentectomy in selected cases and its potential application in more candidates. This approach can be performed successfully with proper localization of the target lung lesion. Further work is required to determine the long-term outcomes and clear benefits of this technique as compared to conventional VATS.

\section{Acknowledgements}

Funding: This work was supported by a grant from the
National Research Foundation of Korea (NRF) funded by the Ministry of Education, Science and Technology (NRF-2015R1A2A2A04005760) and the Korea Health Technology R\&D Project through the Korea Health Industry Development Institute(KHIDI), funded by the Ministry of Health \& Welfare, Republic of Korea (grant number: HR14C0007).

\section{Footnote}

Conflicts of Interest: The authors have no conflicts of interest to declare.

\section{References}

1. Tsutani Y, Miyata Y, Nakayama H, et al. Oncologic outcomes of segmentectomy compared with lobectomy for clinical stage IA lung adenocarcinoma: propensity score-matched analysis in a multicenter study. J Thorac Cardiovasc Surg 2013;146:358-64.

2. Gonzalez-Rivas D, Mendez L, Delgado M, et al. Uniportal video-assisted thoracoscopic anatomic segmentectomy. J Thorac Dis 2013;5 Suppl 3:S226-33.

3. Liu CY, Lin CS, Shih CH, et al. Single-port video-assisted thoracoscopic surgery for lung cancer. J Thorac Dis 2014;6:14-21.

4. Doo KW, Yong HS, Kim HK, et al. Needlescopic resection of small and superficial pulmonary nodule after computed tomographic fluoroscopy-guided dual localization with radiotracer and hookwire. Ann Surg Oncol 2015;22:331-7.

5. Kim HK, Choi YH. The feasibility of single-incision video-assisted thoracoscopic major pulmonary resection performed by surgeons experienced with a two-incision technique. Interact Cardiovasc Thorac Surg 2015;20:310-5.
Cite this article as: Han KN, Kim HK, Choi YH. Uniportal video-assisted thoracoscopic surgical (VATS) segmentectomy with preoperative dual localization: right upper lobe wedge resection and left upper lobe upper division segmentectomy. Ann Cardiothorac Surg 2016;5(2):147-150. doi: 10.21037/ acs.2016.03.06 